\title{
Determinants of Toeplitz-Hessenberg matrices with generalized Fibonacci entries
}

\author{
Taras Goy $^{1}$ and Mark Shattuck ${ }^{2}$ \\ ${ }^{1}$ Faculty of Mathematics and Computer Science \\ Vasyl Stefanyk Precarpathian National University \\ 57 Shevchenko St., 76018 Ivano-Frankivsk, Ukraine \\ e-mail: taras.goy@pnu. edu.ua \\ 2 Department of Mathematics, University of Tennessee \\ 37996 Knoxville, TN, USA \\ e-mail: shattuck@math.utk .edu
}

Received: 19 July 2019

Revised: 10 October 2019

Accepted: 13 October 2019

\begin{abstract}
In this paper, we evaluate several families of Toeplitz-Hessenberg matrices whose entries are generalized Fibonacci numbers. In particular, we find simple formulas for several determinants whose entries are translates of the Chebyshev polynomials of the second kind. Equivalently, these determinant formulas may also be rewritten as identities involving sums of products of generalized Fibonacci numbers and multinomial coefficients. Combinatorial proofs which make use of sign-reversing involutions and the definition of a determinant as a signed sum over the symmetric group $\mathcal{S}_{n}$ are given for our formulas in several particular cases, including those involving the Chebyshev polynomials.
\end{abstract}

Keywords: Toeplitz-Hessenberg matrix, Horadam sequence, Trudi's formula, Generating function, Chebyshev polynomials.

2010 Mathematics Subject Classification: 11B39, 05A15, 15B05.

\section{Introduction}

A generalized Fibonacci sequence $w_{n}=w_{n}(a, b, p, q)$ is one defined by a second order homogeneous linear recurrence

$$
w_{n}=p w_{n-1}+q w_{n-2}, \quad n \geq 2,
$$


with $w_{0}=a$ and $w_{1}=b$, where $a, b, p$ and $q$ are integers with $p>0, q \neq 0$. Since these numbers were first studied by Horadam (see, e.g., [5, 6]), they are often referred to as Horadam numbers. The Horadam numbers are given explicitly [7] by

$$
w_{n}=a \sum_{k=0}^{\left\lfloor\frac{n}{2}\right\rfloor}\left(\begin{array}{c}
n-k \\
k
\end{array}\right) p^{n-2 k} q^{k}+(b-a p) \sum_{k=0}^{\left\lfloor\frac{n-1}{2}\right\rfloor}\left(\begin{array}{c}
n-k-1 \\
k
\end{array}\right) p^{n-2 k-1} q^{k}, \quad n \geq 0 .
$$

In what follows, we will make frequent use of the Binet formula [5] for $w_{n}$ given by

$$
w_{n}=\alpha r_{1}^{n}+\beta r_{2}^{n}, \quad n \geq 0,
$$

where

$$
r_{1}=\frac{p+\sqrt{p^{2}+4 q}}{2}, \quad r_{2}=\frac{p-\sqrt{p^{2}+4 q}}{2}
$$

denote the distinct roots of the quadratic equation $x^{2}-p x-q=0$,

$$
\alpha=\frac{a}{2}+\frac{2 b-a p}{2 \sqrt{p^{2}+4 q}}, \quad \beta=\frac{a}{2}-\frac{2 b-a p}{2 \sqrt{p^{2}+4 q}} .
$$

Note that the parameters $a, b, p$ and $q$ may also be regarded as indeterminates. Further results on Horadam sequences can be found in the survey paper [9].

The sequence $w_{n}$ generalizes many important number and polynomial sequences, for instance, the Fibonacci sequence $F_{n}=w_{n}(0,1,1,1)$, the Lucas sequence $L_{n}=w_{n}(2,1,1,1)$, the Pell sequence $P_{n}=w_{n}(0,1,2,1)$, the Chebyshev polynomials of the first and second kind given by $T_{n}=w_{n}(1, x, 2 x,-1)$ and $U_{n}=w_{n}(1,2 x, 2 x,-1)$, respectively. In [1], Benjamin and Quinn presented combinatorial interpretations for sequences satisfying a recurrence of the form (1) with various initial conditions. For example, $w_{n}$ counts the ways in which to tile an $n$-board with squares representing 1's and dominos representing 2's where each square is assigned the same color as are the dominos except for an initial square or domino (which may be assigned a different color or no color at all).

There is a long tradition of using matrices and determinants to study the Fibonacci numbers and their generalizations. In [14], Tangboonduangjit and Thanatipanonda studied the determinants of matrices whose entries are powers of the Fibonacci numbers, and then extended their results to include entries that are powers of a second-order linear recurrent sequence. İpek [8] computed determinants of several pentadiagonal matrices having generalized Fibonacci and Lucas numbers as entries and also the determinant of a pentadiagonal matrix involving the classical Fibonacci numbers. Applying the apparatus of triangular matrices, Goy [3] established various recurrence formulas for Horadam numbers with even or odd subscripts using determinants of tridiagonal matrices. In [2], employing Hessenberg matrices, Cereceda found some determinantal representations of the general term of a second-order linear recurrent sequence with arbitrary initial values.

The organization of this paper is as follows. In the next section, we find a general determinant formula for Toeplitz-Hessenberg matrices whose entries are Horadam numbers having indices that are consecutive terms of an arbitrary modular class. In the third section, we find some further 
related results involving the Horadam sequence which arise as determinants of certain families of Toeplitz-Hessenberg matrices and particularize these results to Horadam numbers with even or odd subscripts. We also state multi-sum versions of several of our prior formulas which involve products of multinomial coefficients and powers of Horadam numbers. In the final section, we provide combinatorial proofs of several specific cases of the preceding results using the definition of the determinant as a signed sum over $\mathcal{S}_{n}$ and the combinatorial interpretation for $w_{n}$ in terms of tilings.

\section{A general determinant formula}

An $n \times n$ matrix having the form

$$
M_{n}\left(a_{0} ; a_{1}, \ldots, a_{n}\right)=\left[\begin{array}{cccccc}
a_{1} & a_{0} & 0 & \cdots & 0 & 0 \\
a_{2} & a_{1} & a_{0} & \cdots & 0 & 0 \\
a_{3} & a_{2} & a_{1} & \cdots & 0 & 0 \\
\ldots & \ldots & \ldots & \ddots & \cdots & \ldots \\
a_{n-1} & a_{n-2} & a_{n-3} & \cdots & a_{1} & a_{0} \\
a_{n} & a_{n-1} & a_{n-2} & \cdots & a_{2} & a_{1}
\end{array}\right],
$$

where $a_{0} \neq 0$ and $a_{k} \neq 0$ for at least one $k>0$, is called a Toeplitz-Hessenberg matrix. See, e.g., [11] and references contained therein. Expanding the $\operatorname{determinant} \operatorname{det}\left(M_{n}\right)$ along the first row repeatedly, we obtain the recurrence

$$
\operatorname{det}\left(M_{n}\right)=\sum_{k=1}^{n}\left(-a_{0}\right)^{k-1} a_{k} \operatorname{det}\left(M_{n-k}\right), \quad n \geq 1,
$$

where, by definition, $\operatorname{det}\left(M_{0}\right)=1$.

To establish several of our results, we will find a generating function formula for the sequence of determinants in question. Let $f(x)=\sum_{n \geq 1} \operatorname{det}\left(M_{n}\right) x^{n}$.

We will make use of the following result to compute $f(x)$.

Lemma 2.1. We have $f(x)=\frac{g(x)}{1-g(x)}$, where $g(x)=\sum_{k \geq 1}\left(-a_{0}\right)^{k-1} a_{k} x^{k}$.

Proof. Multiplying by $x^{n}$ both sides of the recurrence (3), and summing over all $n \geq 1$, gives

$$
\begin{aligned}
f(x) & =\sum_{n \geq 1} x^{n} \sum_{k=1}^{n}\left(-a_{0}\right)^{k-1} a_{k} \operatorname{det}\left(M_{n-k}\right)=\sum_{k \geq 1}\left(-a_{0}\right)^{k-1} a_{k} \sum_{n \geq k} \operatorname{det}\left(M_{n-k}\right) x^{n} \\
& =\sum_{k \geq 1}\left(-a_{0}\right)^{k-1} a_{k} x^{k} \sum_{n \geq 0} \operatorname{det}\left(M_{n}\right) x^{n}=\sum_{k \geq 1}\left(-a_{0}\right)^{k-1} a_{k} x^{k}(1+f(x))=g(x)(1+f(x)) .
\end{aligned}
$$

Solving for $f(x)$ in the last equation implies the stated formula.

For the sake of brevity, we will write $\operatorname{det}\left(a_{0} ; a_{1}, \ldots, a_{n}\right)$ in place of $\operatorname{det}\left(M_{n}\left(a_{0} ; a_{1}, \ldots, a_{n}\right)\right)$. We have the following general determinant formula for matrices whose entries belong to any modular class $\bmod \ell$ for each $\ell \geq 1$. 
Theorem 2.2. If $n \geq 2, \ell, i \geq 1$ and $0 \leq s \leq \ell-1$, then

$$
\operatorname{det}\left(w_{\ell i+s} ; w_{\ell(i+1)+s}, \ldots, w_{\ell(n+i)+s}\right)=(-1)^{n-1}(-q)^{\ell(n+i-2)+s} d_{\ell} w_{\ell(i-1)+s}^{n-2},
$$

where

$$
d_{\ell}=d_{\ell}(a, b, p, q)=\frac{a^{2} q+a b p-b^{2}}{2^{2 \ell-1}\left(p^{2}+4 q\right)}\left(\sum_{i=0}^{\ell}\left(\begin{array}{l}
2 \ell \\
2 i
\end{array}\right) p^{2 \ell-2 i}\left(p^{2}+4 q\right)^{i}-(-4 q)^{\ell}\right) .
$$

Proof. We first compute

$$
g(x)=\sum_{k \geq 1}\left(-w_{\ell i+s}\right)^{k-1} w_{\ell(i+k)+s} x^{k} .
$$

By the Binet formula for $w_{\ell(i+k)+s}$, we have

$$
\begin{aligned}
g(x) & =\sum_{k \geq 1}\left(-w_{\ell i+s}\right)^{k-1}\left(\alpha r_{1}^{\ell(i+k)+s}+\beta r_{2}^{\ell(i+k)+s}\right) x^{k}=\frac{\alpha r_{1}^{\ell(i+1)+s} x}{1+r_{1}^{\ell} w_{\ell i+s} x}+\frac{\beta r_{2}^{\ell(i+1)+s} x}{1+r_{2}^{\ell} w_{\ell i+s} x} \\
& =\frac{\alpha r_{1}^{\ell(i+1)+s} x\left(1+r_{2}^{\ell} w_{\ell i+s} x\right)+\beta r_{2}^{\ell(i+1)+s} x\left(1+r_{1}^{\ell} w_{\ell i+s} x\right)}{\left(1+r_{1}^{\ell} w_{\ell i+s} x\right)\left(1+r_{2}^{\ell} w_{\ell i+s} x\right)} \\
& =\frac{w_{\ell(i+1)+s} x+w_{\ell i+s}\left(r_{1} r_{2}\right)^{\ell}\left(\alpha r_{1}^{\ell+s}+\beta r_{2}^{\ell i+s}\right) x^{2}}{1+\left(r_{1}^{\ell}+r_{2}^{\ell}\right) w_{\ell i+s} x+\left(r_{1} r_{2}\right)^{\ell} w_{\ell i+s}^{2} x^{2}} \\
& =\frac{w_{\ell(i+1)+s} x+(-q)^{\ell} w_{\ell i+s}^{2} x^{2}}{1+\left(r_{1}^{\ell}+r_{2}^{\ell}\right) w_{\ell i+s} x+(-q)^{\ell} w_{\ell i+s}^{2} x^{2}} .
\end{aligned}
$$

Note that since $i \geq 1$, we have by (2),

$$
\begin{aligned}
& w_{\ell(i+1)+s}-\left(r_{1}^{\ell}+r_{2}^{\ell}\right) w_{\ell i+s}=\alpha r_{1}^{\ell(i+1)+s}+\beta r_{2}^{\ell(i+1)+s}-\left(r_{1}^{\ell}+r_{2}^{\ell}\right)\left(\alpha r_{1}^{\ell i+s}+\beta r_{2}^{\ell i+s}\right) \\
& =-\left(\alpha r_{1}^{\ell i+s} r_{2}^{\ell}+\beta r_{2}^{\ell i+s} r_{1}^{\ell}\right)=-\left(r_{1} r_{2}\right)^{\ell}\left(\alpha r_{1}^{\ell(i-1)+s}+\beta r_{2}^{\ell(i-1)+s}\right)=-(-q)^{\ell} w_{\ell(i-1)+s} .
\end{aligned}
$$

Thus, by Lemma 2.1, we get

$$
\begin{aligned}
f(x) & =\frac{g(x)}{1-g(x)}=\frac{w_{\ell(i+1)+s} x+(-q)^{\ell} w_{\ell i+s}^{2} x^{2}}{1+(-q)^{\ell} w_{\ell(i-1)+s} x} \\
& =w_{\ell(i+1)+s} x+\frac{(-q)^{\ell}\left(w_{\ell i+s}^{2}-w_{\ell(i+1)+s} w_{\ell(i-1)+s}\right) x^{2}}{1+(-q)^{\ell} w_{\ell(i-1)+s} x} .
\end{aligned}
$$

By (2), we have

$$
\begin{aligned}
w_{\ell i+s}^{2}- & w_{\ell(i+1)+s} w_{\ell(i-1)+s} \\
& =\left(\alpha r_{1}^{\ell i+s}+\beta r_{2}^{\ell i+s}\right)^{2}-\left(\alpha r_{1}^{\ell(i+1)+s}+\beta r_{2}^{\ell(i+1)+s}\right)\left(\alpha r_{1}^{\ell(i-1)+s}+\beta r_{2}^{\ell(i-1)+s}\right) \\
& =2 \alpha \beta(-q)^{\ell i+s}-\alpha \beta(-q)^{\ell(i-1)+s}\left(r_{1}^{2 \ell}+r_{2}^{2 \ell}\right) \\
& =\alpha \beta(-q)^{\ell(i-1)+s}\left(2(-q)^{\ell}-\left(r_{1}^{2 \ell}+r_{2}^{2 \ell}\right)\right) .
\end{aligned}
$$

Since $\alpha \beta=\frac{a^{2} q+a b p-b^{2}}{p^{2}+4 q}$ and

$$
r_{1}^{2 \ell}+r_{2}^{2 \ell}=\frac{1}{2^{2 \ell-1}} \sum_{i=0}^{\ell}\left(\begin{array}{l}
2 \ell \\
2 i
\end{array}\right) p^{2 \ell-2 i}\left(p^{2}+4 q\right)^{i}
$$


we then get

$$
f(x)=w_{\ell(i+1)+s} x-\frac{(-q)^{\ell i+s} d_{\ell} x^{2}}{1+(-q)^{\ell} w_{\ell(i-1)+s} x} .
$$

Extracting the coefficient of $x^{n}$ for $n \geq 2$ in the last expression yields the desired formula.

Note that $d_{\ell}$ reduces when $\ell=1$ to $a^{2} q+a b p-b^{2}$, and thus taking $\ell=1$ and $s=0$ in (4) leads to the following result.

Corollary 2.2.1. If $n \geq 2$ and $i \geq 1$, then

$$
\operatorname{det}\left(w_{i} ; w_{i+1}, \ldots, w_{n+i}\right)=(-1)^{i} q^{n+i-2}\left(b^{2}-a b p-a^{2} q\right) w_{i-1}^{n-2} .
$$

The summation within the formula for $d_{\ell}$ reduces when $\ell=2$ to $8 p^{4}+16 q^{2}+32 p^{2} q$, and thus we get $d_{2}=p^{2}\left(a^{2} q+a b p-b^{2}\right)$. Taking $\ell=2$ and $s=0,1$ in (4) then yields the following result.

Corollary 2.2.2. If $n \geq 2$ and $i \geq 1$, then

$$
\operatorname{det}\left(w_{2 i} ; w_{2 i+2}, \ldots, w_{2 n+2 i}\right)=(-1)^{n-1} p^{2} q^{2(n+i-2)}\left(a^{2} q+a b p-b^{2}\right) w_{2 i-2}^{n-2}
$$

and

$$
\operatorname{det}\left(w_{2 i+1} ; w_{2 i+3}, \ldots, w_{2 n+2 i+1}\right)=(-1)^{n} p^{2} q^{2 n+2 i-3}\left(a^{2} q+a b p-b^{2}\right) w_{2 i-1}^{n-2}
$$

\section{Further related formulas}

Let $h(x)=h(x ; c, \ell, s)$ be the generating function given by

$$
h(x)=\sum_{n \geq 1} \operatorname{det}\left(c ; w_{\ell(i+1)+s}, w_{\ell(i+2)+s}, \ldots, w_{\ell(n+i)+s}\right) x^{n}, \quad i \geq 0,
$$

where $c$ is a constant and $\ell$ and $s$ are non-negative integers with $0 \leq s \leq \ell-1$. Proceeding as in the first part of the proof of Theorem 2.2 above, we compute

$$
\begin{aligned}
\sum_{k \geq 1}(-c)^{k-1} w_{\ell(i+k)+s} x^{k}=\sum_{k \geq 1}(-c)^{k-1}\left(\alpha r_{1}^{\ell(i+k)+s}+\beta r_{2}^{\ell(i+k)+s}\right) x^{k} \\
\quad=\frac{w_{\ell(i+1)+s} x+c(-q)^{\ell}\left(\alpha r_{1}^{\ell i+s}+\beta r_{2}^{\ell i+s}\right) x^{2}}{\left(1+c r_{1}^{\ell} x\right)\left(1+c r_{2}^{\ell} x\right)}=\frac{w_{\ell(i+1)+s} x+c(-q)^{\ell} w_{\ell i+s} x^{2}}{1+c\left(r_{1}^{\ell}+r_{2}^{\ell}\right) x+c^{2}(-q)^{\ell} x^{2}} .
\end{aligned}
$$

Applying Lemma 2.1 yields the following formula.

Theorem 3.1. For all $i \geq 0$, we have

$$
h(x)=\frac{w_{\ell(i+1)+s} x+c(-q)^{\ell} w_{\ell i+s} x^{2}}{1+\left(c\left(r_{1}^{\ell}+r_{2}^{\ell}\right)-w_{\ell(i+1)+s}\right) x+c(-q)^{\ell}\left(c-w_{\ell i+s}\right) x^{2}} .
$$

We now state some special cases of the prior result. 
Corollary 3.1.1. If $i \geq 0$, then

$$
h(x ; c, 1,0)=\frac{w_{i+1} x-c q w_{i} x^{2}}{1+\left(c p-w_{i+1}\right) x-c q\left(c-w_{i}\right) x^{2}} .
$$

In particular, we have for $n \geq 2$,

$$
\operatorname{det}\left(w_{0} ; w_{1}, w_{2}, \ldots, w_{n}\right)=\left(b^{2}-a b p-a^{2} q\right)(b-a p)^{n-2}
$$

and

$$
\operatorname{det}\left(w_{1} ; w_{2}, w_{3}, \ldots, w_{n+1}\right)=\left(a^{2} q^{2}+a b p q-b^{2} q\right)(a q)^{n-2} .
$$

Proof. Taking $\ell=1$ and $s=0$ in (5) gives (6). Formulas (7) and (8) follow from letting $c=a, i=0$ and $c=b, i=1$ in (6), respectively, and then extracting the coefficient of $x^{n}$ for $n \geq 2$ in each case.

Remark. Formula (7) is previously known, see [13, Theorem 1], where a different algebraic proof is given which makes use of various matrix decompositions. In the subsequent section, we also provide a combinatorial proof of the $a=1$ and $b=p$ case of (7).

Corollary 3.1.2. If $i \geq 0$, then

$$
h(x ; c, 2, s)=\frac{w_{2 i+s+2} x+c q^{2} w_{2 i+s} x^{2}}{1+\left(c\left(p^{2}+2 q\right)-w_{2 i+s+2}\right) x+c q^{2}\left(c-w_{2 i+s}\right) x^{2}}, \quad s=0,1 .
$$

In particular, we have for $n \geq 2$,

$$
\operatorname{det}\left(w_{0} ; w_{2}, w_{4}, \ldots, w_{2 n}\right)=p^{2}\left(b^{2}-a b p-a^{2} q\right)\left(b p-a q-a p^{2}\right)^{n-2}
$$

and

$$
\operatorname{det}\left(w_{1} ; w_{3}, w_{5}, \ldots, w_{2 n+1}\right)=p^{2} q^{n-1}\left(a^{2} q+a b p-b^{2}\right)(a p-b)^{n-2} .
$$

Proof. Letting $\ell=2$ in (5), and noting $r_{1}^{2}+r_{2}^{2}=p^{2}+2 q$, gives (9). Taking $c=a$ and $i=s=0$ in (9) gives in this case

$$
h(x)=\frac{(a q+b p) x+(a q x)^{2}}{1-\left(b p-a q-a p^{2}\right) x},
$$

which leads to (10). Letting $c=b, i=0$ and $s=1$ in (9) gives

$$
h(x)=\frac{\left(a p q+b q+b p^{2}\right) x+(b q x)^{2}}{1-q(a p-b) x},
$$

which implies (11).

Let $v_{n}=v_{n}(p, q)=w_{n}(1, p, p,-q)$. Then setting $a=1$ and $b=p$ in (7), (8), (10) and (11), replacing $q$ by $-q$, gives respectively

$$
\begin{aligned}
\operatorname{det}\left(v_{0} ; v_{1}, v_{2}, \ldots, v_{n}\right) & =0, \quad n \geq 3, \\
\operatorname{det}\left(v_{1} ; v_{2}, v_{3}, \ldots, v_{n+1}\right) & =(-q)^{n}, \quad n \geq 2, \\
\operatorname{det}\left(v_{0} ; v_{2}, v_{4}, \ldots, v_{2 n}\right) & =p^{2} q^{n-1}, \quad n \geq 2, \\
\operatorname{det}\left(v_{1} ; v_{3}, v_{5}, \ldots, v_{2 n+1}\right) & =0, \quad n \geq 3 .
\end{aligned}
$$


Remark. Note that $U_{n}=v_{n}(2 x, 1)$ for $n \geq 0$, where $U_{n}=U_{n}(x)$ denotes the Chebyshev polynomial of the second kind given by $U_{n}=2 x U_{n-1}-U_{n-2}$ if $n \geq 2$, with $U_{0}=1$ and $U_{1}=2 x$ (see, e.g., [10]). Thus, setting $p=2 x$ and $q=1$ in (12)-(15) yields comparable determinant identities involving Chebyshev polynomials. A similar remark applies to identities (17) and (18) below, upon letting $p=2 x$.

Above we have found determinants of Toeplitz-Hessenberg matrices in which $a_{k}=w_{\ell(i+k)+s}$ for $1 \leq k \leq n$, treating separately the cases when $i>0$ and $i=0$. One can also consider the case when $i=-1$. To do so, let $j(x)=j(x ; c, \ell, s)$ be given by

$$
j(x)=\sum_{n \geq 1} \operatorname{det}\left(c ; w_{s}, w_{\ell+s}, \ldots, w_{\ell(n-1)+s}\right) x^{n} .
$$

Proceeding as before, first note that

$$
\begin{aligned}
& \sum_{k \geq 1}(-c)^{k-1} w_{\ell(k-1)+s} x^{k}=\frac{\alpha r_{1}^{s} x}{1+c r_{1}^{\ell} x}+\frac{\beta r_{2}^{s} x}{1+c r_{2}^{\ell} x}=\frac{\left(\alpha r_{1}^{s}+\beta r_{2}^{s}\right) x+c\left(\alpha r_{1}^{s} r_{2}^{\ell}+\beta r_{1}^{\ell} r_{2}^{s}\right) x^{2}}{\left(1+c r_{1}^{\ell} x\right)\left(1+c r_{2}^{\ell} x\right)} \\
& \quad=\frac{w_{s} x+c\left(\alpha r_{1}^{s} r_{2}^{\ell}+\beta r_{1}^{\ell} r_{2}^{s}\right) x^{2}}{1+c\left(r_{1}^{\ell}+r_{2}^{\ell}\right) x+c^{2}(-q)^{\ell} x^{2}} .
\end{aligned}
$$

Lemma 2.1 then implies the following result.

Theorem 3.2. If $\ell \geq 1$ and $0 \leq s \leq \ell-1$, then

$$
j(x)=\frac{w_{s} x+c\left(\alpha r_{1}^{s} r_{2}^{\ell}+\beta r_{1}^{\ell} r_{2}^{s}\right) x^{2}}{1+\left(c\left(r_{1}^{\ell}+r_{2}^{\ell}\right)-w_{s}\right) x-c\left(\alpha r_{1}^{s} r_{2}^{\ell}+\beta r_{1}^{\ell} r_{2}^{s}-c(-q)^{\ell}\right) x^{2}} .
$$

Let $u_{i}=v_{i}(p, 1)$ and $h_{i}=w_{i}(a, 1,1,-1)$ for $i \geq 0$. Taking $\ell=1$ and $\ell=2$ in (16) yields the following formulas.

Corollary 3.2.1. We have

$$
j(x ; c, 1,0)=\frac{a x+c(a p-b) x^{2}}{1+(c p-a) x-c(a p+c q-b) x^{2}} .
$$

In particular,

$$
\operatorname{det}\left(-u_{1} ; u_{0}, u_{1}, \ldots, u_{n-1}\right)=\sum_{i=0}^{n-1} p^{2 i}, \quad n \geq 1 .
$$

Corollary 3.2.2. We have

$$
j(x ; c, 2,0)=\frac{a x+c\left(a p^{2}+a q-b p\right) x^{2}}{1+\left(c p^{2}+2 c q-a\right) x-c\left(a p^{2}+a q-b p-c q^{2}\right) x^{2}}
$$

and

In particular,

$$
j(x ; c, 2,1)=\frac{b x-c q(a p-b) x^{2}}{1+\left(c p^{2}+2 c q-b\right) x+c q(a p+c q-b) x^{2}} .
$$

$$
\operatorname{det}\left(-u_{0} ; u_{0}, u_{2}, \ldots, u_{2 n-2}\right)=p^{2}\left(p^{2}-1\right)^{n-2}, \quad n \geq 2,
$$

and

$$
\operatorname{det}\left(-h_{1} ; h_{1}, h_{3}, \ldots, h_{2 n-1}\right)= \begin{cases}(-a)^{\frac{n-1}{2}}, & \text { if } n \text { is odd } \\ (1-a)(-a)^{\frac{n-2}{2}}, & \text { if } n \text { is even }\end{cases}
$$


We now give equivalent identities to those above involving multinomial coefficients. The following result, which provides a multinomial expansion of $\operatorname{det}\left(M_{n}\right)$, is known as Trudi's formula, the $a_{0}=1$ case of which is called Brioschi's formula [12].

Lemma 3.3. Let $n$ be a positive integer. Then

$$
\operatorname{det}\left(M_{n}\right)=\left(-a_{0}\right)^{n} \sum_{\left(s_{1}, \ldots, s_{n}\right)}(-1)^{s_{1}+\cdots+s_{n}} p_{n}(s)\left(\frac{a_{1}}{a_{0}}\right)^{s_{1}}\left(\frac{a_{2}}{a_{0}}\right)^{s_{2}} \cdots\left(\frac{a_{n}}{a_{0}}\right)^{s_{n}},
$$

where the summation is over all $n$-tuples of integers $s_{i} \geq 0$ satisfying $s_{1}+2 s_{2}+\cdots+n s_{n}=n$ and $p_{n}(s)=\left(\begin{array}{c}s_{1}+\cdots+s_{n} \\ s_{1}, \ldots, s_{n}\end{array}\right)=\frac{\left(s_{1}+\cdots+s_{n}\right) !}{s_{1} ! \cdots s_{n} !}$ denotes the multinomial coefficient.

The determinant identities above may be rewritten in terms of Trudi's formula as follows.

Theorem 3.4. Let $n \geq 2$, except when noted otherwise, and $i \geq 1$. Then

$$
\begin{aligned}
& \sum_{\left(s_{1}, \ldots, s_{n}\right)}(-1)^{\sigma_{n}} p_{n}(s)\left(\frac{w_{\ell(i+1)+r}}{w_{\ell i+r}}\right)^{s_{1}}\left(\frac{w_{\ell(i+2)+r}}{w_{\ell i+r}}\right)^{s_{2}} \cdots\left(\frac{w_{\ell(i+n)+r}}{w_{\ell i+r}}\right)^{s_{n}} \\
& =-(-q)^{\ell(i+n-2)+r} d_{\ell} \frac{w_{\ell(i-1)+r}^{n-2}}{w_{\ell i+r}^{n}}, \\
& \sum_{\left(s_{1}, \ldots, s_{n}\right)}(-1)^{\sigma_{n}} p_{n}(s)\left(\frac{w_{i+1}}{w_{i}}\right)^{s_{1}}\left(\frac{w_{i+2}}{w_{i}}\right)^{s_{2}} \cdots\left(\frac{w_{i+n}}{w_{i}}\right)^{s_{n}}=\varepsilon(-q)^{i+n-2} \frac{w_{i-1}^{n-2}}{w_{i}^{n}}, \\
& \sum_{\left(s_{1}, \ldots, s_{n}\right)}(-1)^{\sigma_{n}} p_{n}(s)\left(\frac{w_{2 i+2}}{w_{2 i}}\right)^{s_{1}}\left(\frac{w_{2 i+4}}{w_{2 i}}\right)^{s_{2}} \cdots\left(\frac{w_{2 i+2 n}}{w_{2 i}}\right)^{s_{n}}=\varepsilon p^{2} q^{2(i+n-2)} \frac{w_{2 i-2}^{n-2}}{w_{2 i}^{n}}, \\
& \sum_{\left(s_{1}, \ldots, s_{n}\right)}(-1)^{\sigma_{n}} p_{n}(s)\left(\frac{w_{2 i+3}}{w_{2 i+1}}\right)^{s_{1}}\left(\frac{w_{2 i+5}}{w_{2 i+1}}\right)^{s_{2}} \cdots\left(\frac{w_{2 i+2 n+1}}{w_{2 i+1}}\right)^{s_{n}}=-\varepsilon p^{2} q^{2 i+2 n-3} \frac{w_{2 i-1}^{n-2}}{w_{2 i+1}^{n}}, \\
& \sum_{\left(s_{1}, \ldots, s_{n}\right)}(-1)^{\sigma_{n}} p_{n}(s)\left(\frac{w_{1}}{w_{0}}\right)^{s_{1}}\left(\frac{w_{2}}{w_{0}}\right)^{s_{2}} \cdots\left(\frac{w_{n}}{w_{0}}\right)^{s_{n}}=\varepsilon \frac{(a p-b)^{n-2}}{a^{n}}, \\
& \sum_{\left(s_{1}, \ldots, s_{n}\right)}(-1)^{\sigma_{n}} p_{n}(s)\left(\frac{w_{2}}{w_{1}}\right)^{s_{1}}\left(\frac{w_{3}}{w_{1}}\right)^{s_{2}} \cdots\left(\frac{w_{n+1}}{w_{1}}\right)^{s_{n}}=-\varepsilon q \frac{(-a q)^{n-2}}{b^{n}}, \\
& \sum_{\left(s_{1}, \ldots, s_{n}\right)}(-1)^{\sigma_{n}} p_{n}(s)\left(\frac{w_{2}}{w_{0}}\right)^{s_{1}}\left(\frac{w_{4}}{w_{0}}\right)^{s_{2}} \cdots\left(\frac{w_{2 n}}{w_{0}}\right)^{s_{n}}=\varepsilon p^{2} \frac{\left(a p^{2}+a q-b p\right)^{n-2}}{a^{n}}, \\
& \sum_{\left(s_{1}, \ldots, s_{n}\right)}(-1)^{\sigma_{n}} p_{n}(s)\left(\frac{w_{3}}{w_{1}}\right)^{s_{1}}\left(\frac{w_{5}}{w_{1}}\right)^{s_{2}} \cdots\left(\frac{w_{2 n+1}}{w_{1}}\right)^{s_{n}}=-\varepsilon p^{2} q^{n-1} \frac{(b-a p)^{n-2}}{b^{n}} \\
& \sum_{\left(s_{1}, \ldots, s_{n}\right)}(-1)^{\sigma_{n}} p_{n}(s) v_{1}^{s_{1}} v_{2}^{s_{2}} \cdots v_{n}^{s_{n}}=0, \quad n \geq 3 \text {, } \\
& \sum_{\left(s_{1}, \ldots, s_{n}\right)}(-1)^{\sigma_{n}} p_{n}(s)\left(\frac{v_{2}}{v_{1}}\right)^{s_{1}}\left(\frac{v_{3}}{v_{1}}\right)^{s_{2}} \cdots\left(\frac{v_{n+1}}{v_{1}}\right)^{s_{n}}=\left(\frac{q}{p}\right)^{n} \\
& \sum_{\left(s_{1}, \ldots, s_{n}\right)}(-1)^{\sigma_{n}} p_{n}(s) v_{2}^{s_{1}} v_{4}^{s_{2}} \cdots v_{2 n}^{s_{n}}=-p^{2}(-q)^{n-1} \text {, }
\end{aligned}
$$




$$
\begin{aligned}
\sum_{\left(s_{1}, \ldots, s_{n}\right)}(-1)^{\sigma_{n}} p_{n}(s)\left(\frac{v_{3}}{v_{1}}\right)^{s_{1}}\left(\frac{v_{5}}{v_{1}}\right)^{s_{2}} \cdots\left(\frac{v_{2 n+1}}{v_{1}}\right)^{s_{n}} & =0, \quad n \geq 3, \\
\sum_{\left(s_{1}, \ldots, s_{n}\right)} p_{n}(s)\left(\frac{u_{0}}{u_{1}}\right)^{s_{1}}\left(\frac{u_{1}}{u_{1}}\right)^{s_{2}} \cdots\left(\frac{u_{n-1}}{u_{1}}\right)^{s_{n}} & =\frac{1}{p^{n}} \sum_{i=0}^{n-1} p^{2 i}, \quad n \geq 1, \\
\sum_{\left(s_{1}, \ldots, s_{n}\right)} p_{n}(s) u_{0}^{s_{1}} u_{2}^{s_{2}} \cdots u_{2 n-2}^{s_{n}} & =p^{2}\left(p^{2}-1\right)^{n-2},
\end{aligned}
$$

where the summations are over all $n$-tuples $s=\left(s_{1}, s_{2}, \ldots, s_{n}\right)$ of integers $s_{i} \geq 0$ satisfying $s_{1}+2 s_{2}+\cdots+n s_{n}=n, p_{n}(s)=\left(\begin{array}{c}s_{1}+\cdots+s_{n} \\ s_{1}, \ldots, s_{n}\end{array}\right), \sigma_{n}=s_{1}+\cdots+s_{n}, \varepsilon=b^{2}-a b p-a^{2} q$, $v_{m}=v_{m}(p, q)=w_{m}(1, p, p,-q)$ for $m \geq 0, u_{m}=v_{m}(p, 1)$ and $d_{\ell}$ in the first equality is defined as before where $\ell \geq 1$ and $0 \leq r \leq \ell-1$.

Remark. We note that identities five through eight in Theorem 3.4 were announced without proof in [4].

\section{Combinatorial proofs}

In this section, we provide combinatorial proofs of some special cases of the prior results. We first recall the definition of a determinant as a signed sum over the set of permutations. Given an $n \times n$ matrix $A=\left(a_{i, j}\right)$, we have

$$
\operatorname{det}(A)=\sum_{\sigma \in \mathcal{S}_{n}}(-1)^{\operatorname{sgn}(\sigma)} a_{1, \sigma(1)} a_{2, \sigma(2)} \cdots a_{n, \sigma(n)}
$$

where $\operatorname{sgn}(\sigma)$ denotes the sign of the permutation $\sigma$. Suppose that members of $\mathcal{S}_{n}$ are expressed in standard (cycle) form, i.e., where cycles are arranged from left to right in increasing order of their smallest elements, with the smallest element first in each cycle. For a Toeplitz-Hessenberg matrix $A$, the only permutations having non-zero contribution towards the determinant in the sum above are those in which each cycle comprises a set of consecutive integers in increasing order.

Upon identifying the various cycle lengths of such a permutation $\sigma$ expressed in standard form as a sequence of parts, such a $\sigma$ may be regarded as a composition of $n$, which we will denote by $\rho$, where $n$ is the size of $A$. Note that the sign of $\sigma$ is given by $(-1)^{n-(\# \text { of cycles })}$, where the number of cycles of $\sigma$ is the same as the number of parts of $\rho$. So if $A$ is Toeplitz-Hessenberg of size $n$, its determinant may be viewed as a sum of weighted compositions $\rho$ of $n$, where the weight of $\rho$ is the product of the weights of its individual parts times $\left(-a_{0}\right)^{n-m}$, with a part of size $i$ having weight $a_{i}$ for all $i \geq 1$ and $m$ denoting the number of parts of $\rho$.

We will also make use of the combinatorial interpretation for $w_{n}$ in terms of tilings which we now describe. Recall that a (linear) tiling of length $n$ is a covering of the members of $[n]=\{1,2, \ldots, n\}$ written in a row by (indistinguishable) squares and dominos, where a square (domino) covers a single number (resp., two consecutive numbers). We will denote the set of square-and-domino tilings of length $n$ by $\mathcal{T}_{n}$, the members of which we will represent by 
sequences in $\{s, d\}$ such that (\# of $s)+2(\#$ of $d)=n$. Note that $\left|\mathcal{T}_{n}\right|=F_{n+1}$, where $F_{n}$ is the Fibonacci number given by $F_{n}=F_{n-1}+F_{n-2}$ for $n \geq 2$, with $F_{0}=0, F_{1}=1$ (see, e.g., [1, Chapter 1]).

Let us refer to a square or domino which occurs as the first piece within a member of $\mathcal{T}_{n}$ as initial, with all other pieces being non-initial. Individual tiles within a member of $\mathcal{T}_{n}$ are weighted as follows: (i) non-initial squares and dominos receive weights of $p$ and $q$, respectively, and (ii) initial squares and dominos receive weights of $b$ and $a q$, respectively. (Note that when $a=1$ and $b=p$, all squares are assigned weight $p$ and all dominos weight $q$.) Define the weight of a member of $\mathcal{T}_{n}$ as the product of the weights of its individual pieces. Then, it is seen that $w_{n}(a, b, p, q)$ gives the sum of the weights of all members of $\mathcal{T}_{n}$. Note that $w_{n}$ reduces to $F_{n+1}$ when all parameters are unity, the cardinality of the underlying set $\mathcal{T}_{n}$. In the case when $a_{i}=w_{i}$ for all $i$ (or equal some translate of the $w_{i}$ ) in the matrix $A$ above, one may associate with each part of the associated composition $\rho$ a tiling of the same length (or of a length differing from the part size by a fixed amount), weighted as described above. Thus if $\rho$ has $m$ parts, then the weight assigned to $\rho$ is given by the product of the weights of the tilings associated with the various parts of $\rho$ times $\left(-a_{0}\right)^{n-m}$.

We first prove formulas (12) and (17) above where in these cases we consider tilings of length $n$ in which pieces are marked and weighted in various ways.

\section{Bijective proofs of (12) and (17)}

To show (12), first let $\mathcal{A}_{n}$ denote the set of tilings of length $n$ in which any tile may be marked, with the final tile always marked. Squares and dominos (whether marked or not) are assigned weights of $p$ and $-q$, respectively, and define the weight of a member of $\mathcal{A}_{n}$ to be the product of the weights of its pieces and the sign to be $(-1)^{n-(\# \text { of marked tiles) }}$. Since the positions of the marked pieces when taken together yield a composition of $n$ (whose parts are associated with tilings of the same length), we have that $\operatorname{det}\left(v_{0} ; v_{1}, \ldots, v_{n}\right)$ gives the sum of the (signed) weights of all members of $\mathcal{A}_{n}$, by the definition of a determinant. Consider the penultimate tile within a member of $\mathcal{A}_{n}$ (which must exist since $n \geq 3$ ), and either mark the tile or remove the marking from it. This operation defines a sign-changing involution of $\mathcal{A}_{n}$ and $\operatorname{implies} \operatorname{det}\left(v_{0} ; v_{1}, \ldots, v_{n}\right)=0$ for $n \geq 3$.

For (17), let $\mathcal{B}_{n}$ denote the set of tilings of length $n$ in which squares may be marked and the final piece is a marked square. Define the sign of $\lambda \in \mathcal{B}_{n}$ to be $(-1)^{\# \text { of dominos }}$ and the weight of $\lambda$ to be $p^{\mu(\lambda)}$, where

$$
\mu(\lambda)=n+(\# \text { of unmarked squares })-(\# \text { of marked squares }) .
$$

Since compositions of $n$ (wherein a part of size $m$ for each $m$ is associated with a tiling of the length $m-1$ ) may be obtained by considering the positions of the marked squares, we have that the sum of the (signed) weights of all the members of $\mathcal{B}_{n}$ corresponds to $\operatorname{det}\left(-u_{1} ; u_{0}, \ldots, u_{n-1}\right)$. Let $\mathcal{B}_{n}^{*}$ denote the set of all $\rho \in \mathcal{B}_{n}$ of the form $\rho=s^{i}\left(s^{\prime}\right)^{n-i}$, where $0 \leq i \leq n-1$ and $s^{\prime}$ denotes a marked square. The sum of the weights of the members of $\mathcal{B}_{n}^{*}$ is given by $\sum_{i=0}^{n-1} p^{2 i}$. Define an involution of $\mathcal{B}_{n}-\mathcal{B}_{n}^{*}$ by identifying the rightmost position $j$ such that $j, j+1$ is covered by $d$ or $s^{\prime} s$, and switching to the other option. Since this operation preserves the weight (as we are either 
adding or deleting both a marked and an unmarked square), while reversing the sign, the stated determinant formula follows.

\section{Proofs of (14) and (18)}

To show (14), let $\mathcal{C}_{n}$ denote the set of tilings of length $2 n$ where $n \geq 2$ in which a square covering $2 i$ or a domino covering $2 i-1$ and $2 i$ for some $i \in[n]$ may be marked, with the final piece always marked. Define the weights comparably as we did before on $\mathcal{A}_{n}$, with the sign given by $(-1)^{n-(\# \text { of marked tiles) }}$. Since halving the position numbers corresponding to the marked tiles within a member of $\mathcal{C}_{n}$, and taking the differences between the successive numbers so obtained, yields a composition of $n$ (wherein a part of size $m$ for each $m$ is associated with a tiling of length $2 m$ ), it is seen that the sum of the (signed) weights of all members of $\mathcal{C}_{n}$ is given by $\operatorname{det}\left(v_{0} ; v_{2}, \ldots, v_{2 n}\right)$. Define a sign-changing involution of $\mathcal{C}_{n}$ by considering the largest $i \in[n-1]$ such that $2 i$ is either covered by a square or the right half of a domino and either marking or unmarking the square or domino. This operation is defined for all members of $\mathcal{C}_{n}$ except for $\rho=s d^{n-1} s^{\prime}$, which has weight $(-1)^{n-1} \cdot p^{2}(-q)^{n-1}=p^{2} q^{n-1}$.

For (18), let $\mathcal{D}_{n}$ denote the set of tilings of length $2 n$ in which a domino covering $2 i-1,2 i$ for some $i$ may be marked, with the final piece always a marked domino. Let squares each contribute

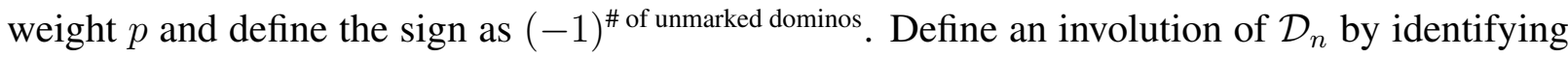
the largest $i \in[n-1]$, if it exists, such that $2 i-1,2 i$ is covered by a domino and either marking or unmarking this domino, which reverses the sign. This involution is not defined on $\mathcal{D}_{n}^{*} \subseteq \mathcal{D}_{n}$ consisting of those tilings in which no consecutive numbers $2 i-1,2 i$ for $i \in[n-1]$ are covered by a domino. Note that $\rho \in \mathcal{D}_{n}^{*}$ implies $\rho=s \rho_{1} \cdots \rho_{n-2} s d^{\prime}$, where each $\rho_{i}=d$ or $s s$. Thus, the sum of the weights of all such members $\rho$ is given by $p^{2}\left(p^{2}-1\right)^{n-2}$, which implies the result.

The bijective proofs provided below of identities (15) and (13) make use of similar arguments involving $m$-tuples of linear tilings for various $m$ whose components have a certain combined length that is now dependent on $m$.

\section{Proofs of (15) and (13)}

To show (15), first let $\mathcal{E}_{n}$ denote the set of sequences $\lambda=\left(\lambda_{1}, \ldots, \lambda_{m}\right)$ of tilings where $m \geq 1$ such that $\sum_{i=1}^{m}\left|\lambda_{i}\right|=2 n+m$, with $\left|\lambda_{i}\right| \geq 3$ odd for all $i$. Define the weight of $\lambda$ to be the product of the weights of each of its component tilings $\lambda_{i}$ (where squares and dominos are assigned weights of $p$ and $-q$, respectively, within each $\lambda_{i}$ ), multiplied by $(-p)^{n-m}$. By the definition of the determinant, we have that $\operatorname{det}\left(v_{1} ; v_{3}, \ldots, v_{2 n+1}\right)$ gives the sum of the weights of all members of $\mathcal{E}_{n}$. Define an involution by pairing members of $\mathcal{E}_{n}$ according to the rightmost component as follows:

(i) $\lambda_{m}=\alpha d \leftrightarrow \lambda_{m}=\alpha, \lambda_{m+1}=s d$,

(ii) $\lambda_{m}=\alpha s^{2} \leftrightarrow \lambda_{m}=\alpha, \lambda_{m+1}=s^{3}$,

(iii) $\lambda_{m}=\alpha d s \leftrightarrow \lambda_{m}=\alpha s, \lambda_{m+1}=d s$,

(iv) $\lambda_{m-1}=\alpha d, \lambda_{m}=d s \leftrightarrow \lambda_{m-1}=\alpha, \lambda_{m}=s d, \lambda_{m+1}=d s$, 
with $|\alpha| \geq 3$ in all cases except for (iii) where $|\alpha| \geq 2$. Since an increase (or decrease) by one in the number of components is always accompanied by the same change in the number of squares, with the number of dominos staying the same, we have that the sign of the weight is always reversed though its magnitude is preserved. Note that case (iv) above requires $n \geq 3$ and hence all members of $\mathcal{E}_{n}$ are paired by (i)-(iv) for $n \geq 3$, which implies (15).

For (13), let $\mathcal{F}_{n}$ for $n \geq 2$ denote the set of sequences $\rho=\left(\rho_{1}, \ldots, \rho_{m}\right)$ of tilings where $m \geq 1$ such that $\sum_{i=1}^{m}\left|\rho_{i}\right|=n+m$, with $\left|\rho_{i}\right| \geq 2$ for all $i$. Define the weight of $\rho$ just as we did for $\lambda$ above. If the final component $\rho_{m}$ within $\rho \in \mathcal{F}$ is not equal to a single $d$, then consider the following pairings where $|\beta| \geq 2$ : (I) $\rho_{m}=\beta s \leftrightarrow \rho_{m}=\beta, \rho_{m+1}=s^{2}$, (II) $\rho_{m}=\beta d \leftrightarrow \rho_{m}=\beta, \rho_{m+1}=s d$. If $\rho_{m}=d$, then look for the largest $i<m$, if it exists, such that $\rho_{i} \neq d$ and perform either of the preceding operations depending on whether the final piece of $\rho_{i}$ is a square or domino. Furthermore, we pair $\left(s^{2}, d, \ldots, d\right)$ with $(s d, d, \ldots, d)$, where the former has $n$ and the latter $n-1$ components. Let $\rho^{\prime}$ denote the member of $\mathcal{F}_{n}$ paired with $\rho$ in each case. Then it is seen that the mapping $\rho \mapsto \rho^{\prime}$ always reverses the sign and preserves the weight, with all members of $\mathcal{F}_{n}$ so paired except for $(d, \ldots, d)$. Thus, we have $\operatorname{det}\left(v_{1} ; v_{2}, \ldots, v_{n+1}\right)=\operatorname{wght}\{(d, \ldots, d)\}=(-q)^{n}$, as desired.

We conclude with a combinatorial proof of formula (19).

\section{Proof of (19)}

Let $\mathcal{G}_{n}$ denote the set of marked tilings of length $2 n$ wherein squares covering an even number may be marked and ending in a marked square. Let $i_{1}<i_{2}<\cdots<i_{k}=2 n$ denote the elements of $[2 n]$ that are covered by a marked square within a member of $\mathcal{G}_{n}$, with $i_{0}=0$. By the $j$-th segment of $\lambda \in \mathcal{G}_{n}$ where $1 \leq j \leq k$, we mean the subcovering within $\lambda$ of the numbers in the set $\left[i_{j-1}+1, i_{j}\right]$. Assign the weight $-a$ to a domino occurring as the first piece within a segment of $\lambda$, with all other dominos and all squares having weights of -1 and 1 , respectively. Then it is seen that $\operatorname{det}\left(-h_{1} ; h_{1}, \ldots, h_{2 n-1}\right)$ gives the sum of the weights of all members of $\mathcal{G}_{n}$ subject to this weighting of tiles.

We now define a sign-reversing involution on $\mathcal{G}_{n}$ as follows. First consider the largest $\ell \in[n-1]$, if it exists, such that the numbers $2 \ell, 2 \ell+1$ are covered by either $d$ or $s^{\prime} s$, where $s^{\prime}$ denotes a marked square, and replace with the other option. If the preceding operation cannot be performed, then consider the largest $\ell \in[n-1]$ such that the numbers $2 \ell-1,2 \ell$ are covered by $d$ or $s s$, where neither the $d$ nor the first $s$ starts some segment, and switch to the other option. Note that one of the preceding operations is always defined if it is the case that a tiling contains a non-initial segment that is not equal to $d s s^{\prime}$ or if the initial segment of a tiling is of length at least six. Thus, if $n$ is odd, the only survivor of the involution above is given by $\alpha=s s^{\prime}\left(d s s^{\prime}\right)^{\frac{n-1}{2}}$. Since $\operatorname{wght}(\{\alpha\})=(-a)^{\frac{n-1}{2}}$, this establishes the odd case of (19). If $n$ is even, there are two survivors of the involution given by $\alpha=\left(d s s^{\prime}\right)^{\frac{n}{2}}$ and $\beta=s^{3} s^{\prime}\left(d s s^{\prime}\right)^{\frac{n-2}{2}}$. Then wght $(\{\alpha, \beta\})=(1-a)(-a)^{\frac{n-2}{2}}$, which implies the even case of (19) and completes the proof. 


\section{References}

[1] Benjamin, A. T. \& Quinn, J. J. (2003). Proofs that Really Count: The Art of Combinatorial Proof, Mathematical Association of America, Washington, DC.

[2] Cereceda, J. L. (2014). Determinantal representations for generalized Fibonacci and Tribonacci numbers, International Journal of Contemporary Mathematical Sciences, 9 (6), 269-285.

[3] Goy, T. (2018). Horadam sequence through recurrent determinants of tridiagonal matrix, Kragujevac Journal of Mathematics, 42 (4), 527-532.

[4] Goy, T. (2019). On some combinatorial identities involving the Horadam numbers, Abstracts of XII International Algebraic Conference in Ukraine, 2-6 July 2019, Vinnytsia, Ukraine, $37-38$.

[5] Horadam, A. F. (1965). Basic properties of a certain generalized sequence of numbers, Fibonacci Quarterly, 3 (2), 161-176.

[6] Horadam, A. F. (1967). Special properties of the sequence $W_{n}(a, b ; p, q)$, Fibonacci Quarterly, 5 (5), 424-434.

[7] Horadam, A. F. (1969). Tschebyscheff and other functions associated with the sequence $W_{n}(a, b ; p, q)$, Fibonacci Quarterly, 7 (1), 14-22.

[8] İpek, A. (2011). On the determinants of pentadiagonal matrices with the classical Fibonacci, generalized Fibonacci and Lucas numbers, Eurasian Mathematical Journal, 2 (2), 60-74.

[9] Larcombe, P. J. (2017). Horadam sequences: a survey update and extension, Bulletin of the Institute of Combinatorics and its Applications, 80, 99-118.

[10] Mason, J. C. \& Handscomb, D. C. (2002). Chebyshev Polynomials, Chapman and Hall/CRC, Boca Raton.

[11] Merca, M. (2013). A note on the determinant of a Toeplitz-Hessenberg matrix, Special Matrices, 1, 10-16.

[12] Muir, T. (1960). The Theory of Determinants in the Historical Order of Development, Vol. 3, Dover Publications, New York.

[13] Shen, S.-Q. \& Liu, W.-J. (2016). Inversion of lower Hessenberg matrix involving classical Horadam numbers, International Journal of Algebra, 10 (7), 343-350.

[14] Tangboonduangjit, A. \& Thanatipanonda, T. (2016). Determinants containing powers of generalized Fibonacci numbers, Journal of Integer Sequences, 19, Article 16.7.1. 